\title{
Is visual representation coloured by desire?
}

People are often told they "view the world through rose-coloured glasses". But do desires in fact change perceptual representations? A new study suggests people not only report observing what they wish was true, but they are more likely to see what they wish was true.

\section{Tali Sharot}

Affective Brain Lab, Department of Experimental Psychology, University College London, London, UK.

Take a look at the photo below of the 2016 U.S. Presidential inauguration (Figure 1). Approximately how many people are in the crowd? Your answer likely depends on your political affiliation. If you support the President you will probably return a higher number, than if you oppose the President. In the words of Harper Lee; 'People generally see what they look for and hear what they listen for' 1 . Two individuals will often report perceiving the same image differently if they hold diverse goals and motives. Many such examples have been documented in the literature. For example, thirst effects the likelihood of reporting the perception of transparency2, which is a characteristic associated with water; a person's decision to climb a hill influences their judgement of its steepness3. What is unclear is whether such motivational influences are in fact indicative of what people see or only of what they report seeing 4 . A fascinating study by Leong, Hughes, Wang and Zaki in Nature Human Behaviour re-examines this decades-old problem5.

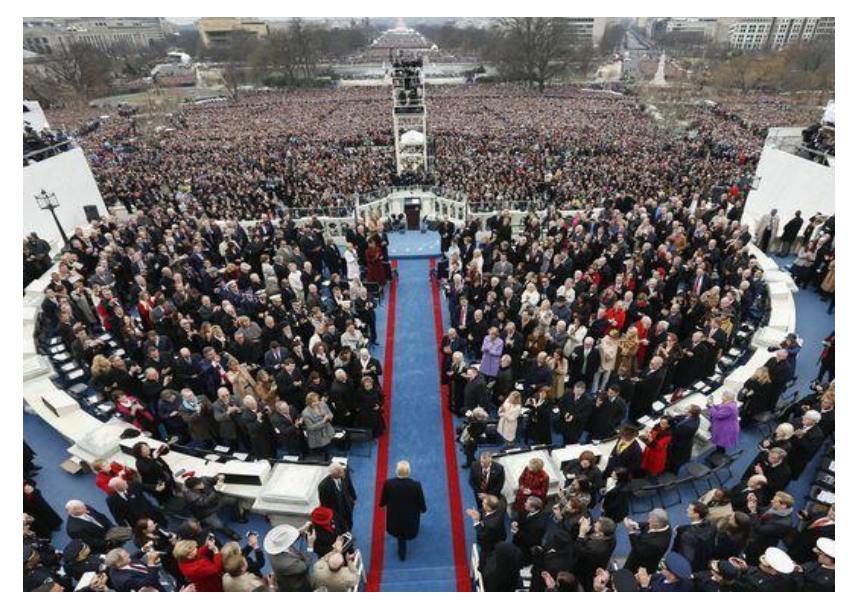

Figure 1. 2016 Presidential Inauguration in Washington DC. Scott Olson / Getty

If motivation alters what people perceive, the authors reason, then corresponding alteration to the pattern of neural activity in visual areas should be observed. If, however, motivation creates a "response bias" than a signature of that modulation should be observed in regions important for motivation before an individual even lays eyes on the stimulus.

To study how motivation alters perception Leong et al., used a task in which participants were presented with an ambiguous image created by morphing a photo of a face with a photo of a house. Participants were rewarded for accurately judging whether the image contained "more 
face" or "more house". On some trials they also received a bonus reward if the stimulus was in fact "mostly house" and on other trials they would receive a bonus if the stimulus was in fact "mostly face". Participants knew in advance which category was most valuable. Importantly, while there was reason to want an image to be "mostly face" or "mostly house", participants could not change the stimulus' category. The only way for them to maximize rewards was to provide accurate responses. Despite this, the motivation to see a particular stimulus biased participants' response towards the desired category, costing them valuable reward points. The results corroborate past findings using a similar approach to study the impact of motivation on perceptual6 and non-perceptual 7 judgements. But does motivation influence the internal visual representation of external stimuli, or are people simply predisposed to report what they wish to be true? The answer, it seems, is both.

First, consistent with a response bias, several seconds before observing the ambiguous stimulus (but after being told which stimulus was most rewarding) BOLD signal in the Nucleus Accumbens (NAcc) was increased on trials in which participants subsequently judged the stimulus to be of the desired category. This finding suggests that NAcc activity predisposes participants to categorize the stimulus as desirable. Indeed, the anticipation of reward is known to trigger activity of dopaminergic neurons that project to the NAcc, invigorating actions associated with those rewards8. As a result, actions that are believed to lead to reward are faster a finding observed also by Leong et al.

Second, the authors found that when faces were the desired category, face-specific activity in the visual stream was stronger, and vice versa for houses. This suggests that the motivation to observe a certain category of stimulus increased the neural representation of that category, presumably altering perceptual experience. One potential explanation is that motivation modulated selective attention. Take the inauguration photo for instance (Figure 1). People may be more likely to attend to the empty spaces at the back if they wish to observe weak support for the President but to the filled spaces in the front if they wish to observe strong support. Different patterns of visual attention will result in different visual input being prioritized by the brain, potentially creating different perceptual experiences. Leong et al., did not record eye movements, which could be analysed in future studies to help determine to what degree selective attention plays a role.

Leong and colleagues contribute to a growing literature showing that a person's desire to believe a certain truth strongly influences how they process information. Such effects include modulation of learning, memory and attention 10 . Because information is processed in a biased manner it is especially difficult to correct motivated beliefs with new evidence, such as correcting ones' scientific theory in light of counter evidence.

Biases in how we perceive the world could also enhance polarization. This is because the same exact event, perceived differently by two individuals, may cause both to become more confident in their conflicting opinions. But research such as that of Leong and colleagues has the potential to facilitate understanding across groups with diverse motives. Knowing that another's visual representation of an event, such as a Presidential Inauguration, can be markedly different than ours, and that neither is an impartial representation of reality, may help us accept other's judgements as genuine.

\section{References}

1. Lee, H. (1988). To kill a mockingbird. Grand Central Publishing. Pg. 174 
2. Changizi, M. A., \& Hall, W. G. (2001). Thirst modulates a perception. Perception, 30(12), 1489-1497.

3. Balcetis, E., \& Dunning, D. (2007). Cognitive dissonance and the perception of natural environments. Psychological Science, 18(10), 917-921.

4. Firestone, C., \& Scholl, B. J. (2016). Cognition does not affect perception: Evaluating the evidence for "top-down" effects. Behavioral and brain sciences, 39.

5. Leong, Y. C., Hughes, B. L., Wang, Y., \& Zaki, J. (2019). Neurocomputational mechanisms underlying motivated seeing. Nature Human Behaviour

6. Voss, A., Rothermund, K. \& Brandtstädter, J. Interpreting ambiguous stimuli: Separating perceptual and judgmental biases. J. Exp. Soc. Psychol. 44, 1048-1056 (2008).

7. Gesiarz F. Cahill, D.P. Sharot T. (in press) Evidence Accumulation is Biased by Motivation: A Computational Account. PLOS Computational Biology

8. Berridge KC, Robinson TE (1998) What is the role of dopamine in reward: Hedonic impact, reward learning, or incentive salience? Brain Res Rev 28:309-369.

9. Sharot, T., Korn, C. W. \& Dolan, R. J. (2011) How unrealistic optimism is maintained in the face of reality. Nature Neuroscience, 14, 1475-1479.

10. Ferrari, V., Codispoti, M., Cardinale, R., \& Bradley, M. M. (2008). Directed and Motivated Attention during Processing of Natural Scenes. Journal of Cognitive Neuroscience, 20(10), 1753-1761.

The authors declare no competing interests. 